\title{
Evaluating Neuromodulator-controlled Stochastic Plasticity for Learning Recurrent Neural Control Networks
}

\author{
Christian W. Rempis, Hazem Toutounji and Frank Pasemann \\ Institute of Cognitive Science, AG Neurocybernetics, Osnabrueck University, 49076 Osnabrueck, Germany
}

\begin{abstract}
Keywords: Neuromodulation, Benchmark, Learning, Sensori-motor Loop, Neurorobotics.
Abstract: Learning recurrent neural networks as behavior controllers for robots requires measures to guide the learning towards a desired behavior. Organisms in nature solve this problem with feedback signals to assess their behavior and to refine their actions. In line with this, a neural framework is developed where the synaptic learning is controlled by artificial neuromodulators that are produced in response to (undesired) sensory signals. To test this framework and to get a base line to evaluate further approaches, we perform five classical benchmark experiments with a simple random plasticity method. We show that even with this simple plasticity method, behaviors can already be found for all experiments, even for comparably large networks with over 90 plastic synapses. The performance depends strongly on the complexity of the task and less on the chosen network topology. This suggests that controlling learning with neuromodulators is a viable approach that is promising to work also with more sophisticated plasticity methods in the future.
\end{abstract}

\section{INTRODUCTION}

One of the major challenges in the field of neurorobotics is to enable a robot to autonomously learn neural networks as behavior controllers in the sensorimotor loop. In this domain, efficient learning methods are rare due to the difficulties inherent to learning in recurrent neural networks. Although some approaches have been reported (Soltoggio and Stanley, 2012; Pitonakova, 2012; Dürr et al., 2008; Hoinville and Hénaff, 2004; Floreano and Urzelai, 2001), the learning usually takes place in very small networks or with network topologies very specifically adapted to the task. One problem is to provide a proper feedback signal, preferably generated by the network itself, to guide the learning towards the desired behavior. This problem can be addressed by extending the neural network with a neuromodulator (NM) layer (Buckley, 2008; Doya, 2002; Fellous and Linster, 1998) that enables the modulation of the learning process as reaction to the observed behavior. A second problem is the difficulty to decide, whether a synaptic weight should be increased or decreased. In contrast to classical feedforward training, neither the desired output, nor the actual effect of a weight change is known. Increasing and decreasing a synapse in such a network may have the same effect on the behavior, depending on the other parts of the network. Hence, the direct- edness of the learning is a problem, because there are no convincing heuristics about the correct direction of a weight change.

To systematically examine these problems, we presented a first framework for feedback driven learning with neuromodulator networks in (Rempis et al., 2013). This framework (Sect. 2) allows the description of a desired behavior in terms of modulator subnetworks, small network structures that monitor the behavior of the robot and stimulate so-called neuromodulator cells (NMCs) in response to undesired or beneficial behavior. Stimulated NMCs produce neuromodulators to trigger or inhibit plastic changes.

In the previous work we could show, that even with a trivial plasticity method - random weight changes - simple behaviors like obstacle avoidance, locomotion and tropisms can be learned successfully from scratch. In accordance with Ashby's ultrastable systems (Ashby, 1960), such networks stabilize in a desired behavior after a while and keep that behavior until a failure triggers further plastic changes to find a better suited network configuration.

In this contribution, we provide a further analysis of the performance and the limitations of this simple approach. For this, we define five benchmark experiments and test the learning performance with different topologies of plastic network structures. These benchmarks, among others, will serve as a base for 
comparing more sophisticated learning approaches currently under development. However, the benchmarks may be of general interest for comparing different approaches against the simple random search to ensure that the methods are, indeed, better.

In the next section we describe the modulator network model with the simple random plasticity method, followed by an introduction of the benchmarks. Then, the results of the learning experiments are discussed, in particular the limitations and characteristics of this learning approach.

\section{METHODS}

A modulated neural network (MNN) can be based on any kind of standard artificial neural network, extended by a neuromodulator layer (Buckley, 2008). Some related approaches, though more specialized, are e.g. GasNets (Husbands, 1998), Artificial Endocrine Systems (Timmis et al., 2009) and Artificial Hormone Systems (Moioli et al., 2009).

Our variant of a NM layer provides neuromodulator cells (NMCs) that maintain spatial distributions of NM concentrations as part of the network. NM produced by a NMC usually diffuses into the surrounding tissue and influences nearby network structures. Each NMC represents a single source for a specific NM type and maintains its own concentration level and distribution within the network. The NM concentration $c(t, x, y)$ at each point in the network at time $t$ is the sum of all locally maintained concentration levels $c_{i}(t, x, y)$ at that position.

NMCs are always in one of two modes: In production mode the cell may increase its modulator concentration, in reduction mode it may decrease it. To enter the production mode, a NMC must be stimulated for some time, whereas it falls back into reduction mode when it was not stimulated for a while. Usually, the concentration of the NM and its area of influence increase and decrease depending on the current stimulation and mode.

\subsection{Linearly Modulated Neural Networks (LMNN)}

The specific variant of the modulated neural network used for the first presented experiments is based on the standard discrete-time neuron model given by

$$
\left.o_{i}(t+1)=\tau_{i}\left(\theta_{i}+\sum_{j=1}^{n} w_{i j} o_{j}(t)\right)\right), \quad i, j=1, \ldots, n
$$

where $o_{i}(t)$ is the output of the neuron $i$ at a discrete time step $t, w_{i j}$ is the weight of the synapse from neuron $j$ to neuron $i, \theta_{i}$ is a bias term of neuron $i$ and $\tau_{i}$ a transfer function, for instance tanh.

The stimulation of NMCs follows a simple linear model. Each NMC is attached to a neuron and is stimulated when the output of this neuron is within a specified range $\left[S^{\min }, S^{\max }\right]$. At each time step $t$, in which the NMC is stimulated, its stimulation level $s_{i}$ increases by a small amount given by parameter $S^{\text {gain }}$. If not stimulated, it decreases by $S^{d r o p}$. If the stimulation level exceeds a given threshold $T^{\text {prod }}$, the NMC enters the production mode. If the level decreases below a second threshold $T^{\text {red }}$, the NMC re-enters the reduction mode. This allows the definition of various delays and hysteresis effects between the two modes, which is an important prerequisite for stable learning (see Sect. 3).

$$
s_{i}(t+1)= \begin{cases}\min \left(1, s_{i}(t)+S_{i}^{\text {gain }}\right) & \text { if } S_{i}^{\text {min }} \leq o_{i}(t) \leq S_{i}^{\max } \\ \max \left(0, s_{i}(t)-S_{i}^{\text {drop }}\right) & \text { otherwise }\end{cases}
$$

In production mode the modulator concentration $c$ and the radius $r$ of, here, a circular diffusion area are increased from 0 to $C^{\max }$ and $R^{\max }$ respectively. During reduction mode both decrease again. The rate of change of the concentration is given by parameters $C^{\text {gain }}$ and $C^{\text {drop }}$, that of the radius similarly by $R^{\text {gain }}$ and $R^{d r o p}$. Equation 3 shows this for the concentration level $c_{i}$; the area radius $r_{i}$ is defined analogously.

$c_{i}(t+1)= \begin{cases}\min \left(C_{i}^{\max }, c_{i}(t)+C_{i}^{\text {gain }}\right) & \begin{array}{l}\text { if production mode } \\ \text { and still stimulated }\end{array} \\ \max \left(0, c_{i}(t)-C_{i}^{d r o p}\right) & \text { if reduction mode } \\ c_{i}(t) & \text { and not stimulated } \\ \text { otherwise }\end{cases}$

The diffusion mode of each NMC can be chosen, so that the NM concentration is either constant across the diffusion area, or decays according to a linear or nonlinear function of the distance to the NMC. The inhomogeneous distributions are interesting for scenarios with local learning. However, in the shown examples, we will restrict the experiments to a homogeneous, global modulation to demonstrate that successful controllers can develop even in this simple case.

\subsection{Plasticity via Modulated Random Search}

The synapses of the network react to NM exposure with plastic changes. To demonstrate the viability of 
Table 1: Parameters of a NMC in a $L M N N$.

\begin{tabular}{l|l}
\hline Parameter & Description \\
\hline Type & The NM type produced by this NMC \\
$S^{\text {min }}, S^{\text {max }}$ & Stimulating neuron activation range \\
$S^{\text {gain }}, S^{\text {drop }}$ & Rate of stimulation gain and drop \\
$T^{\text {prod }}, T^{\text {red }}$ & Pro- and reduction mode thresholds \\
$C^{\text {max }}$ & Max. concentration level of this NMC \\
$C^{\text {gain }}, C^{\text {drop }}$ & Rates of concentration gain and drop \\
$R^{\text {max }}$ & Max. radius of the diffusion area \\
$R^{\text {gain }}, R^{\text {drop }}$ & Rates of diffusion area gain and drop \\
\hline
\end{tabular}

using neuromodulation to control the learning process, we choose one of the most simple plasticity methods available: Random weight changes. We chose this stochastic plasticity method because it is vastly unbiased and is capable of finding all kinds of network topologies and weight distributions within a given network substrate. Furthermore, the method does not require any heuristics for the choice of the network topology, except that solutions are possible with the given structure.

For a synapse $i$, the probability of a weight change $p_{i}^{w}$ at time $t$ is the product of an intrinsic weight change probability $W_{i}$ and the current NM concentration $c(t, x, y)$ at the position $\left(x_{i}, y_{i}\right)$ of the synapse. Hereby, each synapse may limit its sensitivity to NM to a maximal concentration level $M_{i}$ to prevent too rapid changes when large amounts of overlapping NMs are present.

$$
p_{i}^{w}(t)=\min \left(M_{i}, c\left(t, x_{i}, y_{i}\right)\right) W_{i}, \quad 0<W_{i} \lll 1
$$

Stochastic weight changes may occur at any time step, therefore $W_{i}$ must be very small. If a weight change is triggered, a new weight $w_{i}$ is randomly chosen from the interval $\left[W_{i}^{\min }, W_{i}^{\max }\right]$, given as parameters of the synapse.

In addition to weight changes, synapses can also disable and re-enable themselves following a similar stochastic process. The probability $p_{i}^{d}$ for a transition between the two states during each time step is the product of the modulator concentration $c(t, x, y)$ and the disable probability $D_{i}$.

$$
p_{i}^{d}(t)=\min \left(M_{i}, c\left(t, x_{i}, y_{i}\right)\right) D_{i}, \quad 0 \leq D_{i}<W_{i}
$$

If a transition is triggered, an enabled synapse becomes disabled and vice versa. A disabled synapse is treated as a synapse with weight $w_{i}=0$, but its actual weight is preserved until it is enabled again. This mechanism allows for a simple topology search within a given neural substrate.
Table 2: Parameters of a Modulated Random Search synapse.

\begin{tabular}{l|l}
\hline Parameter & Description \\
\hline Type & The NM type the synapse is sensitive to \\
$W$ & Weight change probability \\
$D$ & Disable / enable probability \\
$W^{\min }, W^{\max }$ & Min. and max. weight of the synapse \\
$M$ & Max. NM sensitivity limit of the synapse \\
\hline
\end{tabular}

\section{EXPERIMENTS}

\subsection{Robots, Tasks and Environments}

The experiments use robot systems typical for classical benchmark problems: a differential drive robot (Fig. 1-e) and a simple pendulum (Fig. 1-f). In all cases, motor neurons with an activation range $[-1,1]$ control the desired velocity of the motors. Negative activations are interpreted as backwards rotation. The differential drive robot is equipped with distance sensors (DS) at the front, eight touch sensors (TS), three ambient light sensors (ALS) to measure brightness at three equally distributed positions on the robot, and three directed light sensors (DLS) in the front of the robot to sense the direction towards light sources (with a maximal viewing angle of \pm 90 degrees). For simplicity, light can penetrate obstacles freely. The pendulum is equipped with an angular sensor for the current angle of the pendulum. All experiments have been simulated with the NERD Toolkit (Rempis et al., 2010) and can be replicated with material from our supplementary page.

The first experiment (E1) is a positive light tropism task (Fig. 1-a). Four light sources are distributed in some distance from the corners of a quadratic arena. At any time, only one light source is switched on. Each light source is bright enough to cover the entire arena. When the robot arrives at that light source, it is switched off and a randomly chosen source is switched on.

The second experiment (E2) focuses on an obstacle avoidance task (Fig. 1-b), where the robot has to navigate in a quadratic environment riddled with round objects and sharp corners. The arena also comprises a number of light sources each emitting a different, homogeneous light that allow the robot to recognize different locations and hence to monitor its own exploration behavior.

As a combination of the previous experiments, E3 extends the first experiment with four small obstacles placed with a small asymmetric shift near the four 

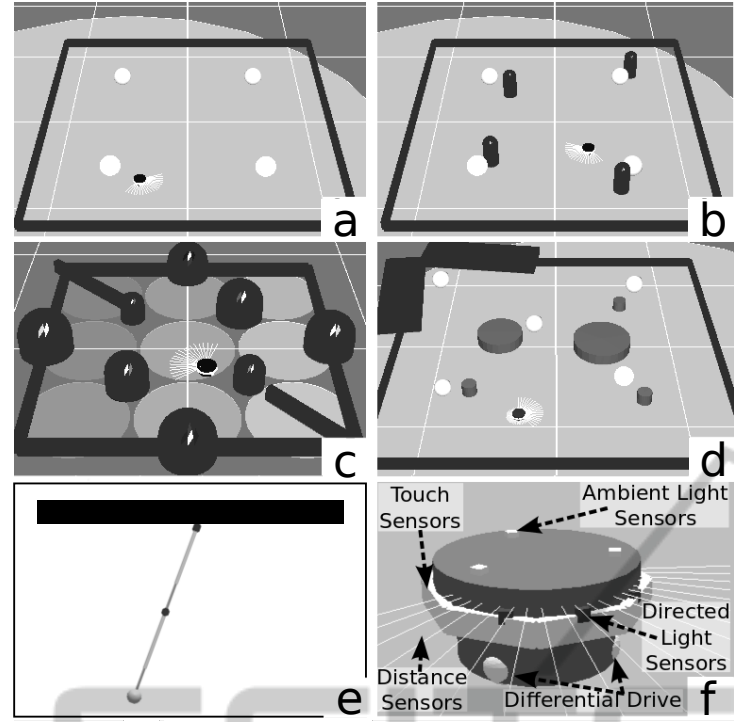

Figure 1: The differential drive robot (f) with three of the environments (a-d) and the simulation of the pendulum (e). The white spheres in $(\mathrm{b}, \mathrm{d})$ denote possible light source positions.

light sources (Fig. 1-c). Here, the robot has to approach the lights and simultaneously avoid the obstacles next to the light sources.

A more difficult variant is experiment $E 4$. While the task remains the same, there are now larger obstacles in the middle of the arena and one of the corners is more narrow (Fig. 1-d). Furthermore, a fifth light source was added in the center of the arena. All lights are now also randomly moved away from their initial positions every time they get switched on. In contrast to E3 the robot now gets confronted with many more different light-obstacle combinations, which makes the task quite difficult.

The pendulum experiment (E5, Fig. 1-e) requires the controller to learn to swing with a specific amplitude between the two target angles $\pm 65^{\circ}$ with a tolerance of $\pm 5^{\circ}$. The difficulty is that the motors are too weak to get to the target angles without swinging the pendulum up first.

\subsection{Control Sub-Networks (CSN)}

Each CSN includes the necessary sensory and motor neurons, a number of intermediate processing neurons and a bias neuron. The latter allows the bias of neurons to be changed using the same technique as used for other synapses. The network substrates vary over the different experiments, ranging from trivial feed-forward networks over a layered network with 4 hidden neurons, to fully connected, recurrent networks with 2, 4 and 6 intermediate neurons. The net-
Table 3: Experiment setups. $\tau_{\exp }$ is the experiment time in simulated minutes, $\tau_{\text {temp }}$ is the duration in minutes without neuromodulation production to consider a behavior a successful temporary solution. See text for further descriptions.

\begin{tabular}{l|l|l|l|l}
\hline Exp. & $\tau_{\exp }$ & $\tau_{\text {temp }}$ & Sensors & NMC Modules \\
\hline E1 & 120 & 0.5 & 2 DLS & Light \\
E2 & 240 & 5 & 3 DS & Obst, Drive, Explore \\
E3 & 720 & 0.75 & 2 DS, 2 DLS & Light, Obst \\
E4 & 720 & 0.75 & 2 DS, 2 DLS & Light, Obst \\
E5 & 240 & 5 & 1 AS & $2 \times$ TurningAngle \\
\hline
\end{tabular}

work configurations for the experiments are summarized in Table 3.

\subsection{Modulatory Sub-Networks (MSN)}

Each MSN uses experiment-specific network structures to detect undesired behavior based on (sensor) activations to produce NMs when needed. As a reaction to the NMs, synapses of the CSN randomly change and explore different topologies and weight distributions. This has an effect on the behavior and, accordingly, on the NM production in the MSN. Similar to the work by Ashby (Ashby, 1960), the system is destabilized when an undesired behavior is detected, leading to continuous changes until the system stabilizes again in a new, valid configuration. In this spirit, six different NMCs are used in the experiments:

The Obst cell reacts on the activation of any of the eight force sensors to detect undesired contact with objects. The stimulation is quite rapid so that obstacle contact immediately leads to NM production to alter the behavior.

Drive gets stimulated when the two motor signals are too low, the robot is moving backwards, or the difference of the motors becomes too large, i.e. the robot is moving in narrow circles. Because the desired behavior also may include moving backwards and especially moving in circles, the stimulation is less rapid and tolerates such movements as long as they do not dominate the behavior.

Explore is stimulated when the robot is not entering the detectable locations frequently. Its associated modulating network classifies the signal of one of the ambient light sensors into the nine detectable locations (inspired by place cells (O'Keefe and Dostrovsky, 1971)) and integrates these signals to determine the duration of each location not being visited. Explore is stimulated if some locations have not been visited for a long time. If a location is entered that has not been visited for a long time, then all integrator neurons for all locations are inhibited, so this potential behavior improvement already leads to a fast NM 

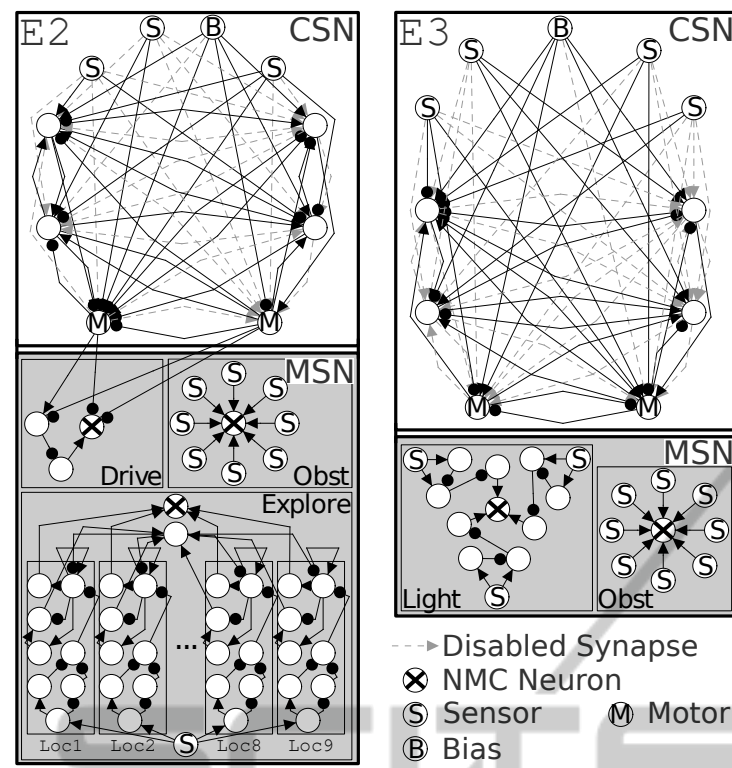

Disabled Synapse

$\boldsymbol{x}$ NMC Neuron

(S) Sensor Motor (B) Bias

Figure 2: Network for Pendulum, Network for Pole Balancer.

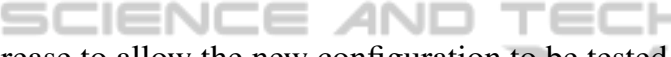

decrease to allow the new configuration to be tested.

The Light cell also uses an auxiliary network that interprets the ambient light sensors to detect whether the robot is getting closer to the light. If not, the NMC is stimulated.

TurningAngle gets persistently, but slowly stimulated over time. However, if the pendulum changes its swinging direction within the desired angle range, then the NMC stimulation decreases rapidly. The desired angular range can be adjusted independently for each of the two NMCs in the pendulum networks.

Table 3 shows which NMCs, with their corresponding auxiliary networks, are used in each experiment. Figure 2 shows the structure of both the $\mathrm{CSN}$ and the MSN for experiments E2 and E3, giving also the neural structures for the six auxiliary subnetworks.

The experiments here are restricted to a global modulator release with a uniform concentration levels. For a discussion, see (Rempis et al., 2013). Table 4 summarizes the parameter choices for the NMCs used across the experiments.

\subsection{Experiments Setup}

Each experiment has been run with five different network substrates for the CSN: a layered network with 4 intermediate neurons ( $\mathrm{L} 4$ ) and four fully, recurrently connected networks with $0,2,4$ and 6 intermediate processing neurons ( $\mathrm{N} 0-\mathrm{N} 6)$. Due to the differing number of motors and sensors, the total number of synapses varies. An overview can be found in table
5. All additional settings of the network, specifically the settings for the plastic synapses and the NMC settings, have been fixed at the values given in tables 4 .

Each such learning scenario (experiment + network substrate) has been repeated 50 times with identical settings, each starting with a new CSN composed of disabled synapses with zero weights. Thus, the entire network topology and the synaptic weights had to be learned from scratch within the given network substrate.

\section{RESULTS AND DISCUSSION}

For all experiments and with all but one of the different network substrates, solutions have been found within the given time windows. All behaviors discovered in this way have been sufficiently effective and comply with the desired and expected behaviors. However, as can be seen in figure 3 , by far not all runs did finally end up with a proper behavior network during the limited learning time. Consistent with intuition, the easier the task is, the larger the percentage of successful learning trials.

The simple light tropism task, therefore, led to successful behaviors in almost all cases, despite its comparably short learning time of up to only two hours. Also, the final solutions have been found very fast (Fig. 4A-E1) without many intermediate temporal solutions (Fig. 4C-E1).

In contrast, the almost similarly short duration of the obstacle avoidance task with four hours seems to be much too low to consistently find solutions, contrary to our expectation. Therefore, only about half of the experiments were successful. A reason for this may be the relatively slow detection of insufficient exploration behavior with the Explore NMC. This modulator has to react with a larger delay to give the networks a chance to actually do exploration. So, behaviors violating the exploration condition - while still doing a fine obstacle avoidance - are detected only after a significant delay. Also, such intermediate solutions get destroyed quite easily when a bad exploration behavior is detected, leading to the destruction - not to a refinement - of the temporary solution. This, obviously, is one of the major limitations of the stochastic search: due to the missing directedness of the learning, temporary solutions are usually not improved, but rather destroyed and replaced by very different networks. This can be seen in figure $4 \mathrm{E}$ that shows the average differences per synapse between two successive (temporary) solutions. Independently of the chosen architecture and the experiment, this difference is quite large with $\approx 0.5$ per synapse 
Table 4: Parameter values for NMCs in the experiments.

\begin{tabular}{l|l|l|l|l|lll|l}
\hline Param. & Obst & Drive & Explore & Light & TurningAngle & & Param. & Synapses \\
\hline$S^{\text {min }}, S^{\text {max }}$ & $0.9,1.0$ & $0.9,1.0$ & $0.4,1.0$ & $0.9,1.0$ & $0.5,1.0$ & & & 0.0001 \\
$S^{\text {gain }}, S^{\text {drop }}$ & $0.01,0.01$ & $0.001,0.001$ & $0.001,0.01$ & $0.0002,0.0001$ & $0.005,1$ & & $D$ & 0.00002 \\
$T^{\text {prod }}, T^{\text {red }}$ & $0.95,0.95$ & $0.95,0.95$ & $0.95,0.95$ & $0.99,0.99$ & $0.95,0.95$ & & $W^{\min }$ & -1.5 \\
$C^{\text {max }}$ & 2 & 1 & 1 & 1 & 1 & $W^{\max }$ & 1.5 \\
$C^{\text {gain }}, C^{\text {drop }}$ & $0.1,0.1$ & $0.001,0.01$ & $0.001,0.01$ & $0.01,0.1$ & $0.001,1$ & & $M$ & 1.0 \\
\hline
\end{tabular}

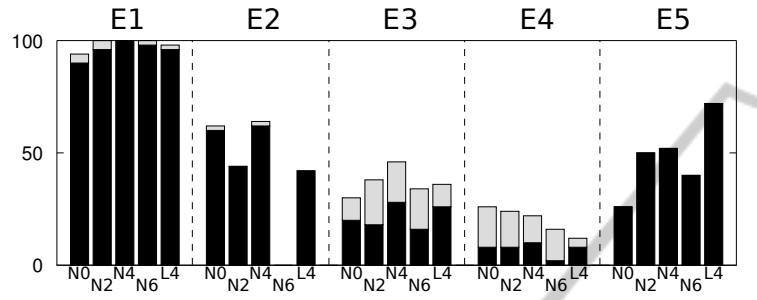

Figure 3: Percentage of successful experiments with stable solutions. The gray tips indicate the number of temporary solutions with a continuous modulator-free behavior during at least 30 minutes, which would be interpreted as solutions in short-term evaluations.

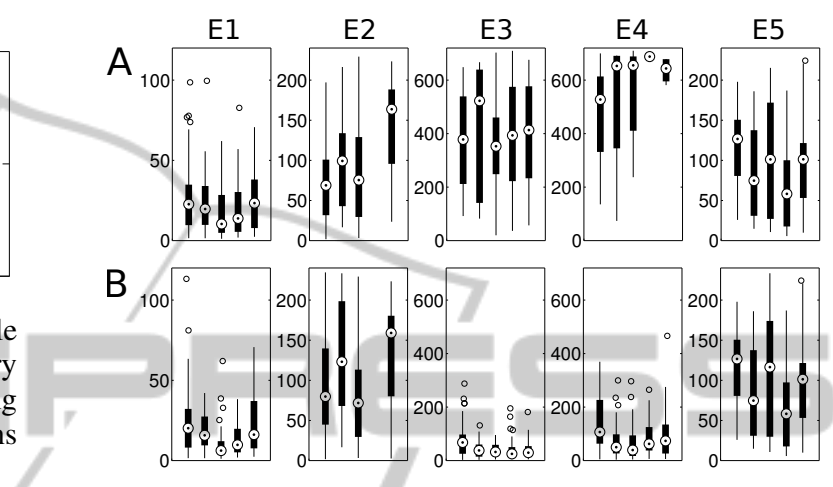

5umber of plastic synapses in each of the experiments. L4 provides a layered network with 4 neurons, all others are fully connected.

\begin{tabular}{l|lllll}
\multicolumn{5}{c}{ Number of Processing Neurons } \\
\cline { 2 - 6 } & N0 & N2 & N4 & N6 & L4 \\
\hline E1 & 14 & 32 & 60 & 96 & 46 \\
E2 & 10 & 28 & 54 & 88 & 42 \\
E3 & 14 & 32 & 60 & 96 & 42 \\
E4 & 14 & 32 & 60 & 96 & 42 \\
E5 & 4 & 15 & 35 & 63 & 32 \\
\hline
\end{tabular}

weight, which indicates large differences between the networks. For the obstacle avoidance behavior this means that large parts of the experimental time are spent with enabled learning (Fig. 4D-E2) or in temporary behaviors that are too short-lived to be considered by us being a temporary solution $(<5$ minutes without modulation, see figure 3 ).

The results for the combination of the two tasks (E3) reflect the increasing difficulty of the task. Even tough the experiment was simulated 12 hours per try, only $\approx 20 \%$ of the runs lead to a fully stable behavior. First temporary solutions have been found quite fast (Fig. 4B-E3), but most light tropism behaviors with only a partial obstacle avoidance behavior are easily destroyed due to hitting one of the small obstacles close to the light sources. Because the light sources are approached with slightly different angles, at some point a situation is encountered where the obstacle avoidance behavior briefly fails and the obsta-

C

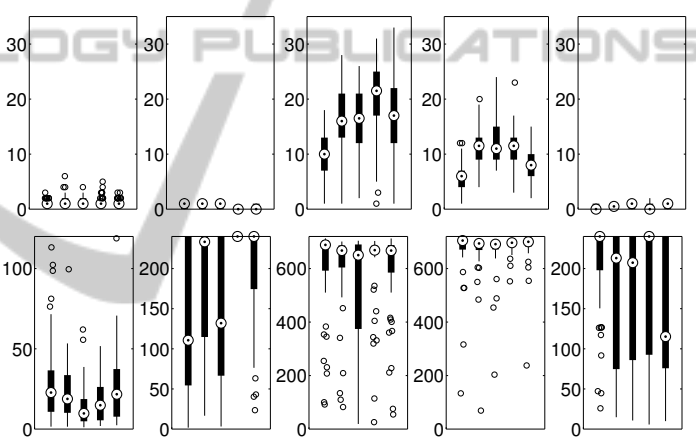

E

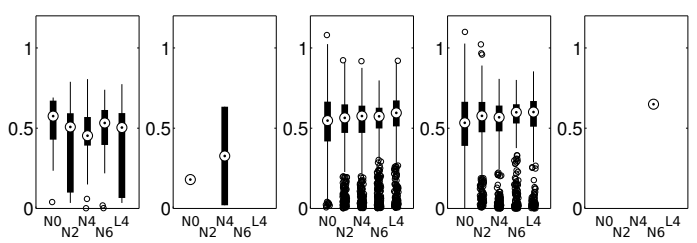

Figure 4: (A) Time to final solution. (B) Time to first (temporary) solution. (C) Number of (temporary) solutions. (D) Minutes spent in learning mode. (E) Average changes per synapse between successive (temporary) solutions.

cle is hit. This leads to a strong production of NM and the behavior is usually destroyed. This alternation between many temporary solutions (Fig. 4C-E3) and the subsequent network destruction, and thus long phases with enabled plasticity (Fig. 4D-E3), describes the typical way how network configurations are explored with the stochastic search: only if all requirements of the behavior are fully met with a single mutation burst, the behavior remains stable in the long run. This all or nothing approach is another limiting characteristics of the simple stochastic search.

This becomes even more severe in the aggravated 


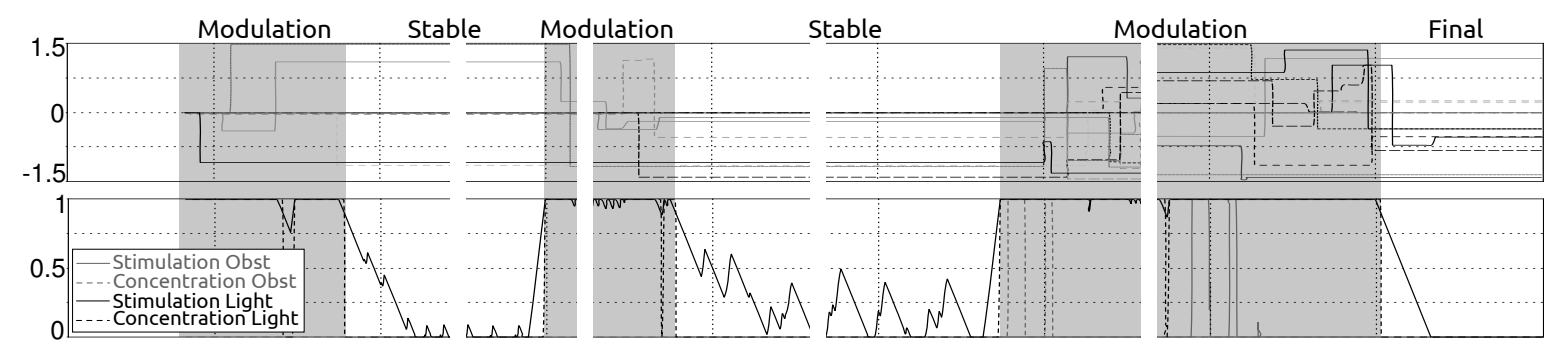

Figure 5: Example run for the light tropism behavior, showing the alternation between stable and plastic states during the behavior learning. The upper graph shows the individual weights over time, the lower graph the stimulation and concentration level of the two NMCs.

variant of this experiment ( $\mathrm{E} 4)$, in which large and more various obstacles enforce the robot to do significant detours against the desired direction towards the light. Here, a proper behavior requires a fine tuning of weights, which makes it much more difficult to accidentally stumble upon a working network. The percentage of final solutions, therefore, is even lower with only about $10 \%$. However, the number of longterm temporary solutions with a continuous runtime of more than 30 minutes exceeds the number of stable solutions by a factor of $\approx 2$ (Fig. 3E4). These behaviors would in many evaluations with a short test (e.g. evolutionary algorithms) already be considered solutions, but it shows that even slight weaknesses due to an unfortunate sequence of target light sources can lead to a destruction of such almost stable networks in the long run. As in E3, temporary solutions are found quite fast (Fig. 4B-E4), but are destroyed later, so that most of the time is spent trying new network configurations (Fig. 4D-E4).

The pendulum behavior again is an example of a simpler single-goal task. The number of successful runs is, with almost $50 \%$, quite high and the networks are also found fast within the first 2 hours (of a total of 4 hours). Due to the characteristics of the experiment, there are almost no temporary solutions: if a solution is found, then this solution tends to be stable in the long run, because there are no disturbances in the simple pendulum motion (compare Fig. 4A-E5, 4B-E5, and 4C-E5).

An interesting observation can be made concerning the network complexity. It was expected, that the performance of the experiments primarily depends on the size of the neural substrate, because with an increasing search space the probability of finding a stable solution should drop down significantly. However, at least for the network sizes used in these experiments, there is only a small influence of the network substrate on the performance (Fig. 3). Only in E2 the largest network showed a significant drop in the number of solutions compared to the other substrates in the same experiment. And in $\mathrm{E} 5$ it seems that the layered network has an advantage over the fully recurrent neural networks. This may indicate, that - as long as the topology can vary within the substrate there are similar or equivalent network configurations contained in all substrates and that with an increasing number of synapses, the fraction between feasible and improper network configurations may remain in the same order of magnitude. In forthcoming experiments, larger networks have to be tested to find the actual limiting size for this simple class of robot experiments. In these experiments, anyway, the impact of the chosen experiment complexity has a much higher impact on the performance than the chosen network substrate, so the major effort in designing such experiments should probably be focused on defining a well suited experiment, not on choosing a particularly suited network substrate.

To examine the learning process in more detail, figure 5 shows the weight changes and the related neuromodulator concentrations for one of the learning runs in experiment E2. As expected, the weight changes in learning phases are random and undirected. However, from time to time, the system stabilizes in a network configuration, because no neuromodulator is produced as a response to the (partially) working behavior. It can also be seen in the lower part of figure 5 that even during these stable states, the stimulation of the NMCs is not just zero, but that their stimulation level remains active, though not high enough to enter their production mode. So, slight violations of the behavior restrictions still take place, but these violations are not strong enough to be interpreted as a failing behavior. But if the stimulation level exceeds the limit to production mode, then often one of the first random changes destabilizes the system so much, that other neuromodulators are triggered as side-effect. This leads to a strong relearning, usually destroying the previous temporary solution, until the modulation stops when a new potentially working configuration has been found. 


\section{CONCLUSIONS}

We demonstrated with five typical experiments from the field of robot learning and early evolutionary robotics, that a simple random search on a given network topology is sufficient to find many suitable solutions, as long as the network changes are started and stopped by a reasonable feedback signal. In our case, this feedback is realized with neuromodulators that are triggered as a reaction to the sensed behavior. Because of this, and the simplicity of the implementation, the learning should also work directly on physical robots without external supervision. The benchmarks show that the feasibility of the method strongly depends on the experiment complexity, not so much on the chosen network substrate. Also, temporary solutions appear and get relearned when the behavior proves ineffective in some situations. These aspects - already available in such a simple approach - are highly desired in the field of robot learning to allow adaptive, self-contained robots with life-long learning capabilities. The method, however, is not meant to be used as a competitive learning paradigm for real robots. Instead, one intention of the benchmark is to provide a minimal testbed to evaluate new learning paradigms for recurrent neural networks in the sensori-motor loop. These paradigms should be better in some aspects compared to such a simple random search to justify their usually much higher complexity. For this, the benchmarks are also publicly available at the supplementary page.

\section{Supplementary Material can be found at:}

nerd.x-bot.org/neuromodulator-benchmarks

\section{ACKNOWLEDGEMENTS}

This work was partially funded by DFG-grant PA 480/7-1. We thank Josef Behr and Florian Ziegler for testing and refining the simulation model and for their contributions to the NERD toolkit.

\section{REFERENCES}

Ashby, W. R. (1960). Design for a brain: The origin of adaptive behavior (2nd edition). Chapman and Hall, London UK.

Buckley, C. L. (2008). A systemic analysis of the ideas immanent in neuromodulation. $\mathrm{PhD}$ thesis, University of Southampton.
Doya, K. (2002). Metalearning and neuromodulation. Neural Networks, 15(4-6):495-506.

Dürr, P., Mattiussi, C., Soltoggio, A., and Floreano, D. (2008). Evolvability of neuromodulated learning for robots. In Proc. of the 2008 ECSIS Symposium on Learning and Adaptive Behavior in Robotic Systems, pages $41-46$.

Fellous, J. and Linster, C. (1998). Computational models of neuromodulation. Neural Computation, 10(4):771805.

Floreano, D. and Urzelai, J. (2001). Neural morphogenesis, synaptic plasticity, and evolution. Theory in Biosciences, 120(3):225-240.

Hoinville, T. and Hénaff, P. (2004). Comparative study of two homeostatic mechanisms in evolved neural controllers for legged locomotion. In Proc. of IEEE/RSJ International Conference on Intelligent Robots and Systems, IROS 2004, volume 3, pages 2624-2629.

Husbands, P. (1998). Evolving robot behaviours with diffusing gas networks. In Proc. of Evolutionary Robotics, EvoRob'98, volume 1468 of LNCS, pages 71-86. Springer.

Moioli, R., Vargas, P., and Husbands, P. (2009). A multiple hormone approach to the homeostatic control of conflicting behaviours in an autonomous mobile robot. In Proc. of IEEE Congress on Evolutionary Computation, CEC'09, pages 47-54.

O'Keefe, J. and Dostrovsky, J. (1971). The hippocampus as a spatial map: Preliminary evidence from unit activity in the freely-moving rat. Brain Research, 34(1):171175.

Pitonakova, L. (2012). Ultrastable neuroendocrine robot controller. Adaptive Behavior, 21(1):47-63.

Rempis, C. W., Thomas, V., Bachmann, F., and Pasemann, F. (2010). NERD - Neurodynamics and Evolutionary Robotics Development Kit. In SIMPAR 2010, volume 6472 of $L N A I$, pages 121-132. Springer.

Rempis, C. W., Toutounji, H., and Pasemann, F. (2013). Controlling the learning of behaviors in the sensorimotor loop with neuromodulators in self-monitoring neural networks. In Workshop on Autonomous Learning at the IEEE International Conference on Robotics and Automation, ICRA 2013.

Soltoggio, A. and Stanley, K. (2012). From modulated hebbian plasticity to simple behavior learning through noise and weight saturation. Neural Networks, 34:2841.

Timmis, J., Neal, M., and Thorniley, J. (2009). An adaptive neuro-endocrine system for robotic systems. In Proc. of the IEEE Workshop on Robotic Intelligence in Informationally Structured Space, RIISS'09, pages 129-136. 